\title{
Response time measurement in flow induced signal generation on semiconductors
}

\author{
Sankha S. Sarkar ${ }^{\mathrm{a}, 1}$, S. Ghosh ${ }^{\mathrm{b}, *}$, A.K. Sood ${ }^{\mathrm{a}, \mathrm{c}, \mathrm{d}}$ \\ a Department of Physics, Indian Institute of Science, Bangalore 560012, India \\ ${ }^{\mathrm{b}}$ Department of Condensed Matter Physics and Material Sciences, Tata Institute of Fundamental Research, Mumbai 400005, India \\ ${ }^{\mathrm{c}}$ Chemistry and Physics of Materials Unit, Jawaharlal Nehru Centre for Advanced Scientific Research, Bangalore 560064, India \\ ${ }^{\mathrm{d}}$ Unit on Nanoscience and Technology DST, IISc and JNCASR, Bangalore, India \\ Received 27 June 2006; received in revised form 26 February 2007; accepted 28 February 2007 \\ Available online 3 March 2007
}

\begin{abstract}
Measurable electrical signal is generated when a gas flows over a variety of solids, including doped semiconductors, even at the modest speed of a few meters per second. The underlying mechanism is an interesting interplay of Bernoulli's principle and the Seebeck effect. The electrical signal depends on the square of Mach number $(M)$ and is proportional to the Seebeck coefficient $(S)$ of the solids. Here we present experimental estimate of the response time of the signal rise and fall process, i.e. how fast the semiconductor materials respond to a steady flow as soon as it is set on or off. A theoretical model is also presented to understand the process and the dependence of the response time on the nature and physical dimensions of the semiconductor material used and they are compared with the experimental observations.
\end{abstract}

(C) 2007 Elsevier B.V. All rights reserved.

Keywords: Seebeck effect; Bernoulli’s principle; Response time; Semiconductor; Heat diffusion

\section{Introduction}

The recent finding on the phenomenon of flow induced voltage generation on semiconductors [1] is an interesting observation in terms of basic physics and its potential applications. It was reported that a gas flow at modest speed of few meters per second over a variety of solids generates finite voltages across the solid sample. These classes of solids include single and multiwalled carbon nanotubes, graphite and doped semiconductors. The underlying mechanism is a nice interplay of Bernoulli's principle and the Seebeck effect.

When a steady flow passes over an inclined surface, the flow velocity $(v(x))$ along with the length $(x)$ on the surface measured from the leading edge varies as a power-law in $x, v(x) \sim x^{m}$, where the index $m$ depends upon the angle $(\theta)$ of inclination

\footnotetext{
* Corresponding author. Tel.: +91222278 2314; fax: +91 2222804610.

E-mail addresses: sankha.sarkar@psi.ch (S.S. Sarkar), sghosh@mailhost.tifr.res.in (S. Ghosh), asood@physics.iisc.ernet.in (A.K. Sood)

${ }^{1}$ Present address: Laboratory for Micro- and Nanotechnology, Paul Scherrer Institute, 5232 Villigen PSI, CH, Switzerland.
}

(for $\theta=45^{\circ}, m=1 / 3$ ) [2]. The velocity difference between the leading edge and the rare end on the solid surface produces a pressure difference $(\Delta P)$ due to Bernoulli's principle. Pressure difference along a streamline over an inclined plane gives rise to temperature differences $(\Delta T)$ across the sample due to the equation of state in incompressible gases $(\triangle P / P=\Delta T / T, P$ and $T$ being the absolute pressure and temperature, respectively). This temperature difference produces a finite voltage $(V)$ due to Seebeck effect, $V=S \Delta T, S$ being the difference in the Seebeck coefficients of the semiconductor and the metal with which the electrical contacts are made. The electrical signal depends on the Mach number $M$ as $V \sim M^{2}$, where $M=u / c_{\mathrm{s}}, u$ is the gas flow velocity and $c_{\mathrm{S}}$ is the sound velocity in the gas.

In the present work, we report the measurement on the response time of the flow induced signal generated across the semiconductor materials to a steady flow. The response time is a measure of the promptness of the flow induced signal to follow the onset or offset of the steady flow. This is an important parameter in terms of the flow-sensor design. Here, we present an estimate for the response time and its dependence on the geometry and material property of the semiconductor used, followed by a theoretical understanding of the process. 


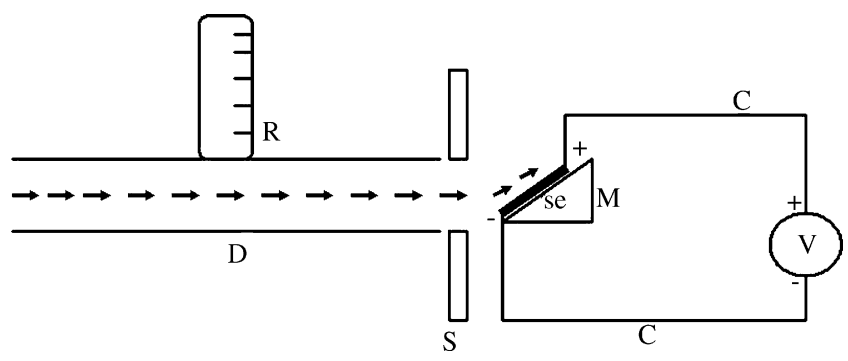

Fig. 1. Schematic of the experimental setup. se: sensor material, M: metallic mount, C: connecting wires, V: multimeter, S: shutter, D: flow duct, R: flowmeter. The arrows show the flow velocity.

\section{Experimental details and results}

The experimental setup [Fig. 1] consists of a flow duct (D) which is connected to a Nitrogen Gas cylinder. The flow rate is measured with the help of a flowmeter (R). The sensor (se) attached to a metal mount $(\mathrm{M})$ is placed in front of the open end of the flow duct at an inclination of $45^{\circ}$. The sensor se is separated from the metal mount $\mathrm{M}$ with electrically and thermally insulating adhesive material. A mechanical shutter $(\mathrm{S})$ is placed between the flow duct and the sensor to set the flow on and off. The shutter response time is calculated to be $<30 \mathrm{~ms}$. The connecting wires (C) are soldered to the two ends of the sensor material (se) with indium and connected to the Keithley 2000 multimeter. The data acquisition rate is $100 \mathrm{~Hz}$. Experiments are done with semiconductors of different physical dimensions (length $(L) \times$ width $(w) \times$ thickness $(l)$ ), e.g. $n$-type Germa$\operatorname{nium}(\mathrm{Ge})(6 \mathrm{~mm} \times 3 \mathrm{~mm} \times 1.2 \mathrm{~mm}$, conductivity $\sigma=10 / \Omega-\mathrm{cm}$, $S=-548 \mu V / K), n$-type Silicon $(\mathrm{Si})(6 \mathrm{~mm} \times 3 \mathrm{~mm} \times 0.6 \mathrm{~mm}$, $\sigma=100 / \Omega-\mathrm{cm}, S=-450 \mu \mathrm{V} / K)$ and $n$-type Bismuth Tellunde $(n$ $\left.\mathrm{Bi}_{2} \mathrm{Te}_{3}, \sigma=1000 / \Omega-\mathrm{cm}, S=-190 \mu V / K\right)($ area $5 \mathrm{~mm} \times 4 \mathrm{~mm}$ ) samples of three different thicknesses $(l=0.95,1.2$ and $1.5 \mathrm{~mm})$. In these sets of experiments, only $n$-type semiconductors are chosen but the flow induced electrical signal generation is observed both on $n$-type and $p$-type semiconductors and are reported in Ref. [1].

The mechanical shutter sets the flow on and off when required. The rise and the fall of the signal voltage is measured with time as soon as the flow is set on and off, respectively. Typical data obtained from the experiment on $\mathrm{Bi}_{2} \mathrm{Te}_{3}$ sample is shown in Fig. 2 (for rise) and Fig. 3 (for fall).

\section{Theoretical model}

The theoretical understanding behind the growth and the decay process of the signal voltage is due the heat conduction from the gas to the solid. Fig. 4 shows the schematic of the flow and temperature profile on the semiconductor when the flow is set on. The temperature $(T)$ of the flow in steady state near point $\mathrm{A}$ and $\mathrm{B}$ are given by $T_{\mathrm{A}}^{F}\left(v_{\mathrm{A}}\right)$ and $T_{\mathrm{B}}^{F}\left(v_{\mathrm{B}}\right)$, respectively. Area ' $a$ ' on the top surface is the region where the electrical contact is made and is assumed to be same for the contact at both ends A and $\mathrm{B}$. The thickness of the semiconducting material is $l$. When the flow is set on, the solid comes in contact with the temperature gradient generated in the flow due to the velocity gradient

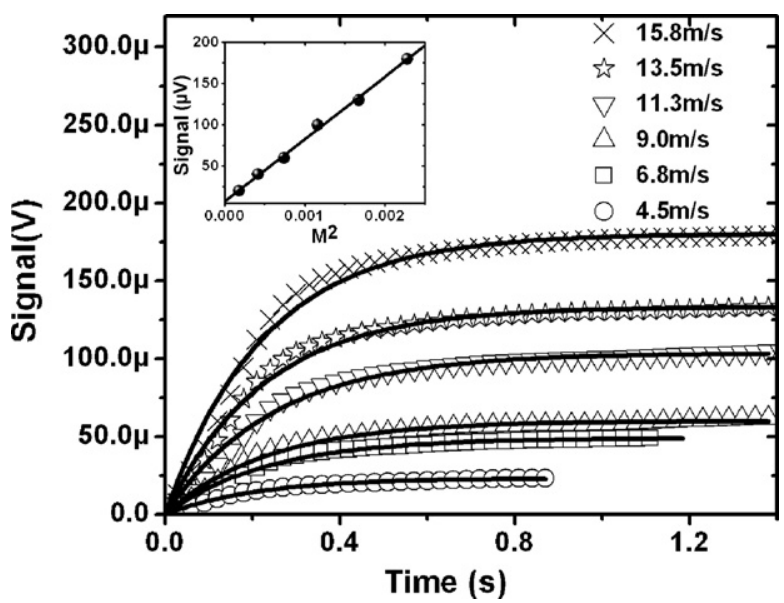

Fig. 2. The rise in the signal in a $\mathrm{Bi}_{2} \mathrm{Te}_{3}(l=1.2 \mathrm{~mm})$ sample is plotted against time for different mean flow velocities. The legend that represents each dataset for different flow velocities is described in the figure. The solid lines are fit to Eq. (7) to respective dataset. The inset shows the fitting parameter $V_{0}$ plotted against $M^{2}$.

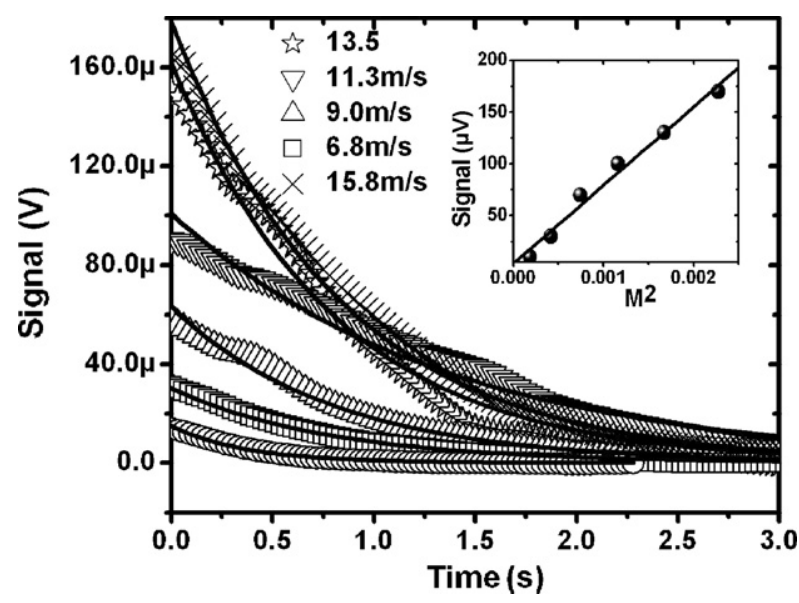

Fig. 3. The fall in the signal in a $\mathrm{Bi}_{2} \mathrm{Te}_{3}(l=1.2 \mathrm{~mm})$ sample is plotted against time for different mean flow velocities. The legend that represents each dataset for different flow velocities is described in the figure. The solid lines are fit to Eq. (10) to respective dataset. The inset shows the fitting parameter $V_{0}$ plotted against $M^{2}$.

$(v(x))$ and the gas acts as a temperature bath for the solid. The temperature in the solid rises and comes in equilibrium with the flow temperature. As the Seebeck voltage is generated due to the temperature difference $(\Delta T)$ at two ends of the solid, the follow-

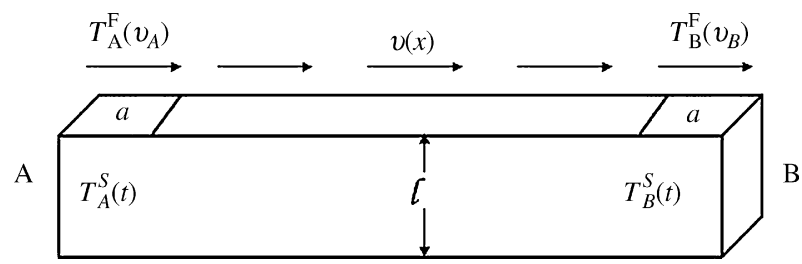

Fig. 4. Schematic of the sensor material under steady flow from $A$ to $B$ (arrows). Temperatures at different regions are shown in the figure. $T_{\mathrm{A}}^{F}\left(v_{\mathrm{A}}\right)\left(T_{\mathrm{B}}^{F}\left(v_{\mathrm{B}}\right)\right)$ is the temperature of the flow near end $\mathrm{A}(\mathrm{B})$ for a velocity $v_{\mathrm{A}}\left(v_{\mathrm{B}}\right) . T_{\mathrm{A}}^{S}(t)\left(T_{\mathrm{B}}^{S}(t)\right)$ is the temperature of the solid at time $t$ at end $\mathrm{A}(\mathrm{B}) . v(x)$ is the velocity profile on the solid as a function of length, $x$. 
ing model only takes into account the temperature rise process at two ends of the solid.

The temperature $(T)$ inside the material at end A and B varies with time $(t)$ and they are given by $T_{\mathrm{A}}^{S}(t)$ and $T_{\mathrm{B}}^{S}(t)$, respectively. $T_{\mathrm{A}}^{S}(t)$ is given by the heat conduction equation

$\rho l a C \frac{\mathrm{d} T_{\mathrm{A}}^{S}(t)}{\mathrm{d} t}=-\kappa_{1} \lambda a \frac{\mathrm{d} T_{\mathrm{A}}^{S}(t)}{\mathrm{d} l}$

where $\rho l a$ is the mass of the material at end A, $\rho$ is the density of the material, $C$ is the specific heat of the material, $\lambda$ is the thermal conductivity of the solid, $\kappa_{1}$ is the coupling factor which takes care of the fact that a certain fraction of the heat that enters into the material is used for the temperature increase and the remaining part is diffused over the rest of the solid. For a small temperature gradient across the gas and the solid interface, it is assumed to be linear as

$\frac{\mathrm{d} T_{\mathrm{A}}^{S}(t)}{\mathrm{d} l} \approx \frac{T_{\mathrm{A}}^{S}(t)-T_{\mathrm{A}}^{F}\left(v_{\mathrm{A}}\right)}{l}$

with this assumption Eq. (1) simplifies into

$\frac{\mathrm{d} T_{\mathrm{A}}^{S}(t)}{\mathrm{dt}}=-\frac{\kappa_{1} \lambda}{\rho l^{2} c}\left[T_{\mathrm{A}}^{S}(t)-T_{\mathrm{A}}^{F}\left(v_{\mathrm{A}}\right)\right]=-\frac{\kappa_{1} h}{l^{2}}\left[T_{\mathrm{A}}^{S}(t)-T_{\mathrm{A}}^{F}\left(v_{\mathrm{A}}\right)\right]$

where $h$ is the thermal diffusivity of the material $(h=\lambda / \rho c)$. Solution to Eq. (3) with the initial condition $T_{\mathrm{A}}^{S}(t=0)=T_{\mathrm{amb}}$ ( $T_{\mathrm{amb}}$ being the ambient temperature without flow) gives the temperature rise profile at end $\mathrm{A}$ as

$T_{\mathrm{A}}^{S}(t)=T_{\mathrm{A}}^{F}\left(v_{\mathrm{A}}\right)-\left[T_{\mathrm{A}}^{F}\left(v_{\mathrm{A}}\right)-T_{\mathrm{amb}}\right] \mathrm{e}^{-\kappa_{1} h t / l^{2}}$

similarly temperature at end B rises as

$T_{\mathrm{B}}^{S}(t)=T_{\mathrm{B}}^{F}\left(v_{\mathrm{B}}\right)-\left[T_{\mathrm{B}}^{F}\left(v_{\mathrm{B}}\right)-T_{\mathrm{amb}}\right] \mathrm{e}^{-\kappa_{1} h t / l^{2}}$

temperature difference between two ends $\mathrm{A}$ and $\mathrm{B}$ rises as

$T_{\mathrm{A}}^{S}(t)-T_{\mathrm{B}}^{S}(t)=\left[T_{\mathrm{A}}^{F}\left(v_{\mathrm{A}}\right)-T_{\mathrm{B}}^{F}\left(v_{\mathrm{B}}\right)\right]\left(1-\mathrm{e}^{-\kappa_{1} h t / l^{2}}\right)$

hence, the Seebeck voltage developed across the material is

$V(t)=k S\left[T_{\mathrm{A}}^{F}\left(v_{\mathrm{A}}\right)-T_{\mathrm{B}}^{F}\left(v_{\mathrm{B}}\right)\right]\left(1-\mathrm{e}^{-\kappa_{1} h t / l^{2}}\right)=V_{0}\left(1-\mathrm{e}^{t / \tau_{1}}\right)$

where $k$ is a coupling factor which depends upon the specific interaction between the flow and the solid surface $[1,3] ; V_{0}$, the steady state response voltage of the sensor is $k S\left[T_{\mathrm{A}}^{F}\left(v_{\mathrm{A}}\right)-\right.$ $\left.T_{\mathrm{B}}^{F}\left(v_{\mathrm{B}}\right)\right]$ and $\tau_{1}=l^{2} / h \kappa_{1}$ is the time constant of the exponential rise process. $\tau_{1}$ is considered as the response time of the process (rise time). The first order exponential rise function Eq. (7) is fitted to each set of data recorded at different flow velocities in Fig. 2.

Similar treatment is done for the fall in signal voltage when the steady flow velocity is set off. Fig. 5 shows the temperature profile on the semiconductor when the flow is set off. The temperature on the surface of the solid comes to the ambient temperature and the solid loses excess heat to the ambience. With

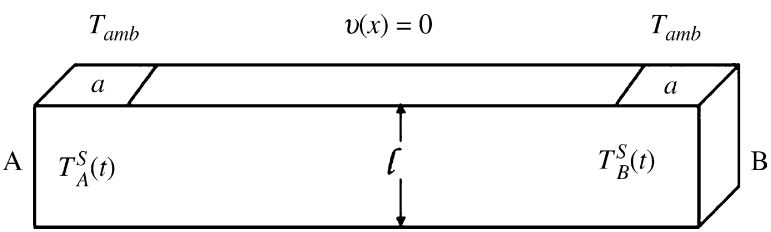

Fig. 5. Schematic of the sensor material and temperatures at different region are shown in the figure when the flow is set off. $T_{\mathrm{A}}^{S}(t)\left(T_{\mathrm{B}}^{S}(t)\right)$ is the temperature of the solid at time $t$ at end A(B). $T_{\mathrm{amb}}$ is the ambient temperature over the solid surface when the flow velocity $(v(x))$ is zero.

an assumption similar to Eq. (2) the heat conduction equation is written as

$-\rho l a C \frac{\mathrm{d} T_{\mathrm{A}}^{S}(t)}{\mathrm{d} t}=-\kappa_{2} \lambda a \frac{T_{\mathrm{A}}^{S}(t)-T_{\mathrm{amb}}}{l}$

where $\kappa_{2}$ is a coupling factor similar to $\kappa_{1}$. The solution to Eq. (8) with the necessary initial condition $T_{\mathrm{A}}^{S}(t=0)=T_{\mathrm{A}}^{F}\left(v_{\mathrm{A}}\right)$ is given as

$T_{\mathrm{A}}^{S}(t)=T_{\mathrm{amb}}+\left[T_{\mathrm{A}}^{F}\left(v_{\mathrm{A}}\right)-T_{\mathrm{amb}}\right] \mathrm{e}^{-\kappa_{2} h t / l^{2}}$

Hence, the Seebeck voltage developed across the solid is given by

$V(t)=k S\left[T_{\mathrm{A}}^{F}\left(v_{\mathrm{A}}\right)-T_{\mathrm{B}}^{F}\left(v_{\mathrm{B}}\right)\right] \mathrm{e}^{-\kappa_{2} h t / l^{2}}=V_{0} \mathrm{e}^{-t / \tau_{2}}$

$\tau_{2}=l^{2} / h \kappa_{2}$ is a quantity similar to $\tau_{1}$ and is considered as the response time of the process (fall time).

\section{Conclusions}

Measurements are done for the rise time on $n$-Ge, $n$-Si, $n$ $\mathrm{Bi}_{2} \mathrm{Te}_{3}$ and for the fall time on $n-\mathrm{Bi}_{2} \mathrm{Te}_{3}$ of three different thicknesses. Fig. 6 shows the values of the rise time and fall time for different materials used at different flow velocities. In Fig. 2 and Fig. 3 , the inset shows the parameter $V_{0}$ obtained from

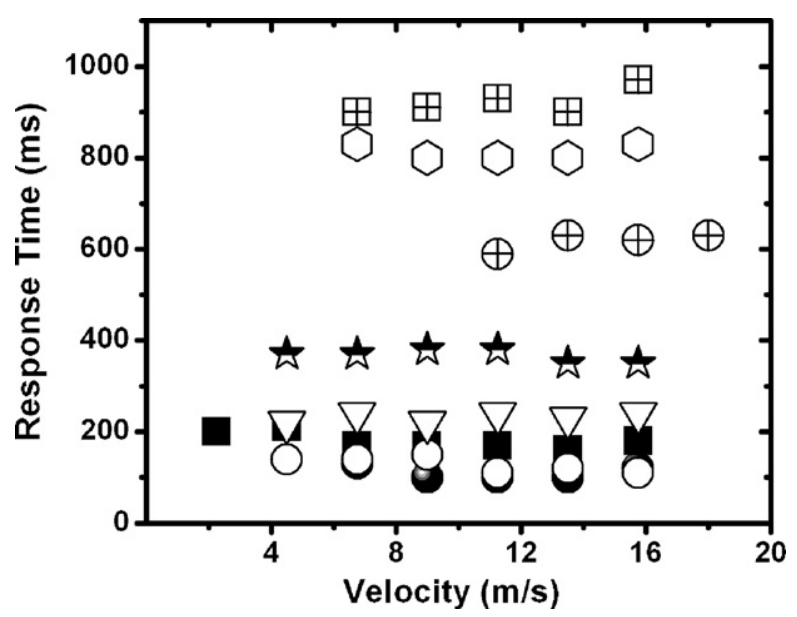

Fig. 6. Response time for different materials at different velocities. Rise time $\left(\tau_{1}\right)$ half shaded circle: $\mathrm{Bi}_{2} \mathrm{Te}_{3} \quad(1=0.95 \mathrm{~mm})$; inverted triangle: $\mathrm{Bi}_{2} \mathrm{Te}_{3}(1=1.2 \mathrm{~mm})$; half shaded star: $\mathrm{Bi}_{2} \mathrm{Te}_{3}(1=1.5 \mathrm{~mm})$; open circle: $\mathrm{Ge}$ $(1=1.2 \mathrm{~mm})$; solid circle: $\mathrm{Si}(1=0.6 \mathrm{~mm})$; fall time $\left(\tau_{2}\right)$ circle with cross: $\mathrm{Bi}_{2} \mathrm{Te}_{3}$ $(1=0.95 \mathrm{~mm})$; open hexagon: $\mathrm{Bi}_{2} \mathrm{Te}_{3}(1=1.2 \mathrm{~mm})$; square with cross: $\mathrm{Bi}_{2} \mathrm{Te}_{3}$ $(1=1.5 \mathrm{~mm})$. 
Table 1

Material parameters and measured response time

\begin{tabular}{lllllll}
\hline Material & $h\left(\mathrm{~m}^{2} / \mathrm{s}\right)[4]$ & $l\left(\times 10^{-3} \mathrm{~m}\right)$ & $\tau_{1}(\mathrm{~s})$ & $\tau_{2}(\mathrm{~s})$ & $\kappa_{1}$ & $\kappa_{2}$ \\
\hline$n-\mathrm{Ge}$ & $3.5 \times 10^{-5}$ & 1.2 & 0.14 & - & 0.29 & - \\
$n-\mathrm{Si}$ & $0.9 \times 10^{-5}$ & 0.6 & 0.11 & - & 0.36 & - \\
$n-\mathrm{Bi}_{2} \mathrm{Te}_{3}$ & $2.9 \times 10^{-5}$ & 0.95 & 0.18 & 0.63 & 0.17 & 0.05 \\
$n-\mathrm{Bi}_{2} \mathrm{Te}_{3}$ & $2.9 \times 10^{-5}$ & 1.2 & 0.23 & 0.81 & 0.20 & 0.06 \\
$n-\mathrm{Bi}_{2} \mathrm{Te}_{3}$ & $2.9 \times 10^{-5}$ & 1.5 & 0.37 & 0.94 & 0.21 & 0.08 \\
\hline
\end{tabular}

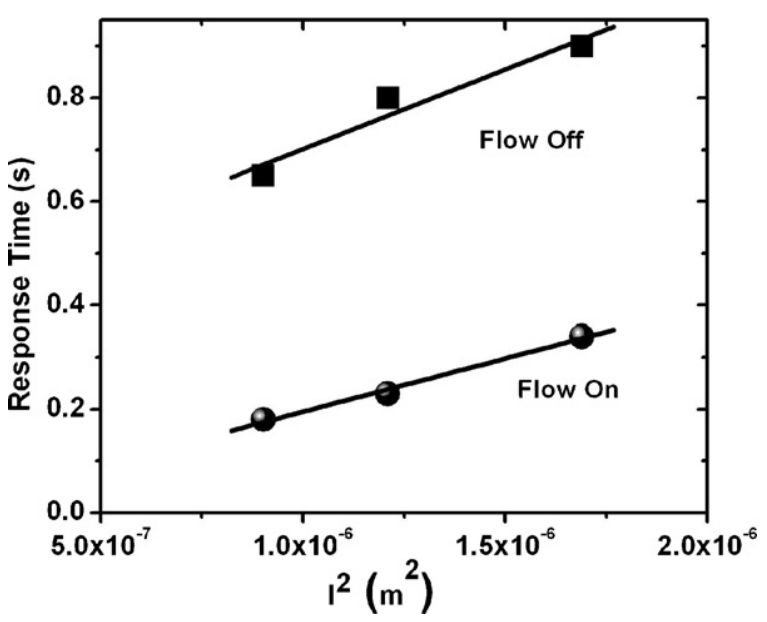

Fig. 7. Variation of $\tau_{1}$ and $\tau_{2}$ with $l^{2}$. Solid circle and square represents the values of $\tau_{1}$ and $\tau_{2}$, respectively. The solid lines are the linear fit to the respective data.

the fitting of Eq. (7) and Eq. (10) to the respective dataset plotted against $M^{2}$ and dependence is linear as expected from Eq. (1). Table 1 shows the values of $\tau_{1}$ and $\tau_{2}$ for different materials and hence the values of $\kappa_{1}$ and $\kappa_{2}$ are calculated which are in good agreement with each other for three $n-\mathrm{Bi}_{2} \mathrm{Te}_{3}$ samples of different thickness. From the theoretical analysis it is expected that the response time is dependent on the thickness of the solid as $\tau_{1} \sim l^{2}$ and $\tau_{2} \sim l^{2}$. The measured values of $\tau_{1}$ and $\tau_{2}$ for three different thicknesses of $n-\mathrm{Bi}_{2} \mathrm{Te}_{3}$ are plotted against $l^{2}$ (Fig. 7) and the linear dependence is evident.

The agreement between the experimental results and the theory outlined above gives an understanding of the temperature, hence signal rise and fall process across the semiconductor mate- rial. It also gives an estimate for the response timescale of the materials. The rise and the fall processes are first order exponential processes. The theoretical model predicts that the response time is inversely proportional to the thermal diffusivity $(h)$ of the material. The rise time for a given material is always less than the fall time. Thickness of the material has a crucial role in determining the response time and the dependence is shown in Fig. 7 which is also supported by the theoretical model.

\section{Acknowledgements}

We would like to thank Dr. O.N. Ramesh and Prof. P. Nott for useful discussions. A.K.S. thanks Department of Science and Technology, India, for financial support.

\section{References}

[1] A.K. Sood, S. Ghosh, Phys. Rev. Lett. 93 (2004) 086601.

[2] H. Schlichting, Boundary Layer Theory, Pergamon Press Ltd., London, 2004, p. 119.

[3] L.D. Landau, E.M. Lifshitz, Physical Kinetics, Butterworth Heine-mann, 1998, p. 51.

[4] D.R. Lide (Ed.), CRC Handbook of Chemistry and Physics, 79th ed., CRC Press, London, 1999.

\section{Biographies}

Sankha S. Sarkar has done his bachelor degree in physics from Jadavpur University, Kolkata, India in 2003. He then joined Indian Institute of Science, Bangalore, India for his Masters degree in physics. The work presented here is done during his Masters project. He is presently in Paul Scherrer Institute, Villigen, Switzerland and working on Extreme Ultraviolet Lithography and soft X-ray microscopy. He has applied to Swiss Federal Institute of Technology (ETH), Zurich for his doctoral work.

S. Ghosh has done his $\mathrm{PhD}$ degree from the Indian Institute of Science, Bangalore in 2005. His PhD work mainly comprised electrical signals generated by flowing fluids over carbon nanotubes. He has now joined Tata institute of Fundamental research, Mumbai, India as a faculty (2005).

A.K. Sood is a professor of physics at Indian Institute of Science, Bangalore, India. His research interests include Physics of Carbon nanotubes including liquid flow induced voltage generation, Raman spectroscopy and electronic transport. He also has made notable contribution in soft condensed matter like chaotic dynamics in flow of micellar gels, colloidal liquids, etc. 